\title{
Topology Optimization OF HoRizontal Crossbeam of Portal Milling Machine
}

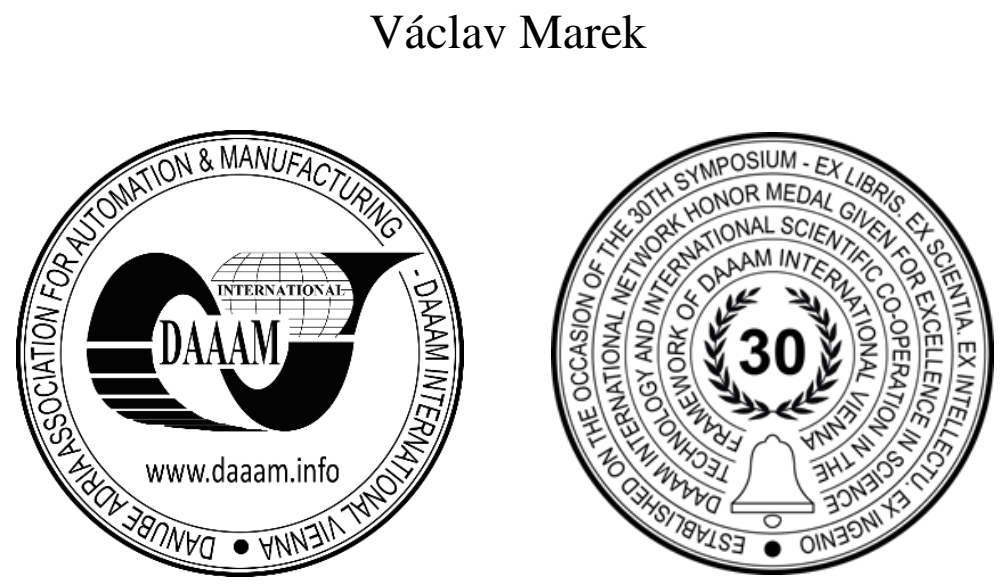

This Publication has to be referred as: Marek, V[aclav] (2019). Topology Optimization of Horizontal Crossbeam of Portal Milling Machine, Proceedings of the 30th DAAAM International Symposium, pp.0586-0591, B. Katalinic (Ed.), Published by DAAAM International, ISBN 978-3-902734-22-8, ISSN 1726-9679, Vienna, Austria DOI: $10.2507 / 30$ th.daaam.proceedings.079

\begin{abstract}
Displacements caused by cutting forces, gravity or mass inertia influent the overall precision and productivity of milling machines. Economic pressure leads to cost reduction of milling centres. Reduction of weights should decrease primematerial costs of milling center. However, non-conception material reduction lead to stiffness descent, problems with harmonic frequencies or technological obstruction. Precise material distribution reduce weight and conserve the stiffness of crossbeams. Through this approach the best structure of loaded part is identified with respect to combination of weight and low compliance. On one hand, optimization identifies the best solution for required, specific boundary condition, but on the other hand it causes lower performance in other unspecified circumstances of loading. Due to that, a precise definition of boundary condition was crucial. Horizontal crossbeam of portal milling center was optimized. Part was optimized isolated from overall assembly. Boundary conditions which define connection between crossbeam and columns was defined with respect to real connection. Hydrostatic guidance between column and crossbeam provides specific connection. It requires precise definition because of the crucial influence on calculation. At the same time, minimal deformation of hydrostatic surfaces is required. Complex topology optimization leads to better material utilization, costeffective production and higher performance of machining.
\end{abstract}

Keywords: final element analysis; topology optimization; milling center

\section{Introduction}

Production precision and performance of milling centres is a vital issue. Topology optimization should be used in early phase of concept design. Topology optimization identifies the best material distribution with respect to boundary conditions and weight reduction. This tool allows to develop feasible, efficient design [1] [2] The Final element analysis, the computed fluid dynamic and other predicting tools are strong tools which require cooperation between the designer and analysis designer. This approach leads to increase of the stiffness and the performance of machining. Precise review of modern commercial optimization software was referred by [3]

Modern loaded structures use bionic design for optimal load distribution [4]. Biomimetic structures are inspired by natureevolution shapes, which represent years of evolution. This approach is an equivalent of the topology optimization. Biomimetic [5] principles are mostly used for highly loaded structures. Inspiration in the bio-structures may identify good 
approach in the beginning of the design. Topology-based structures use similar approach to structural bionic methodology [6].

These methods are also suitable for heat conductivity identification and optimal heat distribution, which is necessary for precision of milling machines [7] [8].The production accuracy of machine tools is effected by the thermo-mechanical behaviour of the components and structural design. Heat distribution and temperature is changed in time due to the timedependent internal and external heat sources [9]. These approaches are suitable and profitable for design of heavy machines [10] [11].

Topology optimization approach is applied on the structural design of a crossbeam of a portal milling centre. Structural design has to reflect stiffness and mass requirement. Variety of loading options defines the loading cases which are applied in the topology setup. This study solves methodology of boundary-condition definition which strongly influents solution of topology optimization. Work also introduce possible interpretation of results. Required structure solution consist of welded sheet.

\section{Topology setup}

In general optimization algorithms give the best possible solutions under given circumstances. Algorithm works with the objective function which defines required property (stiffness, Eigen frequencies etc.) It searches for the maximum/ minimum of the function. [12] [13] [1] [14] Presented case is solved by minimizing the compliance function.

Checkerboard problem is a complication connected with the mesh element size and topology algorithm which lead to generation of checkerboard patterns [12] [15] A few approaches may decrease this influence. These are higher orders of used elements, filter or different discretisation of FE model [15].

Penalty function SIMP was used with penalty parameter 3. Objective function compliance was used because the minimal compliance of structure is required. Minimal value of this function is reached during the computation.

Evaluation of critical boundary condition between subcases is performed by across subcase function MAX/SSQ. Compliance was reduced by design constrains which define the maximal required value. Design constrain of maximal displacement was used. This boundary condition was defined in the tool position. Maximal displacement was defined to be $0.1 \mathrm{~mm}$. Design constrain of total weight was stated to be maximal $76000 \mathrm{~kg}$. This value consists of preserved area and presumed, topology reinforced area. Requirement of minimal deformation of guidance surfaces is $<0.02 \mathrm{~mm}$, according to production prescription. Symmetric manufacturing constrain was used because of the symmetric real work condition. Hydrostatic guidance was defined by Nastran 1D element - cbush 1D element which consists of controlled stiffness. This represents the compliance of a hydraulic oil film. Final element model uses linear CTETRA4 elements for fixed part and cubic linear CHEXA8 elements for optimized area. Final FE model consist of 5milions elements based on elements with size $50 \mathrm{~mm}$. Preliminary calculations were performed with size of elements $80 \mathrm{~mm}$ and $100 \mathrm{~mm}$.

\subsection{Load cases}

Variety of load cases is necessary for the suitable results [12]. Optimized structure for specific cases may cause lower load capacity in the unspecified cases [16]. It may cause failure of parts or technological problems during the production. Two positions of head stock were included to subcases. It is simulated by two positions of loads. Predicted weight of the headstock is stated to be $20000 \mathrm{~kg}$. Bought positions are eccentric. Cutting force is $40 \mathrm{kN}$, predicted in all directions. It is important to emphasise that loading with full cutting force with requirement of low deformation is critical, exceptional, atypical case. Finish milling operations with precision requirements are not performed with full cutting force.
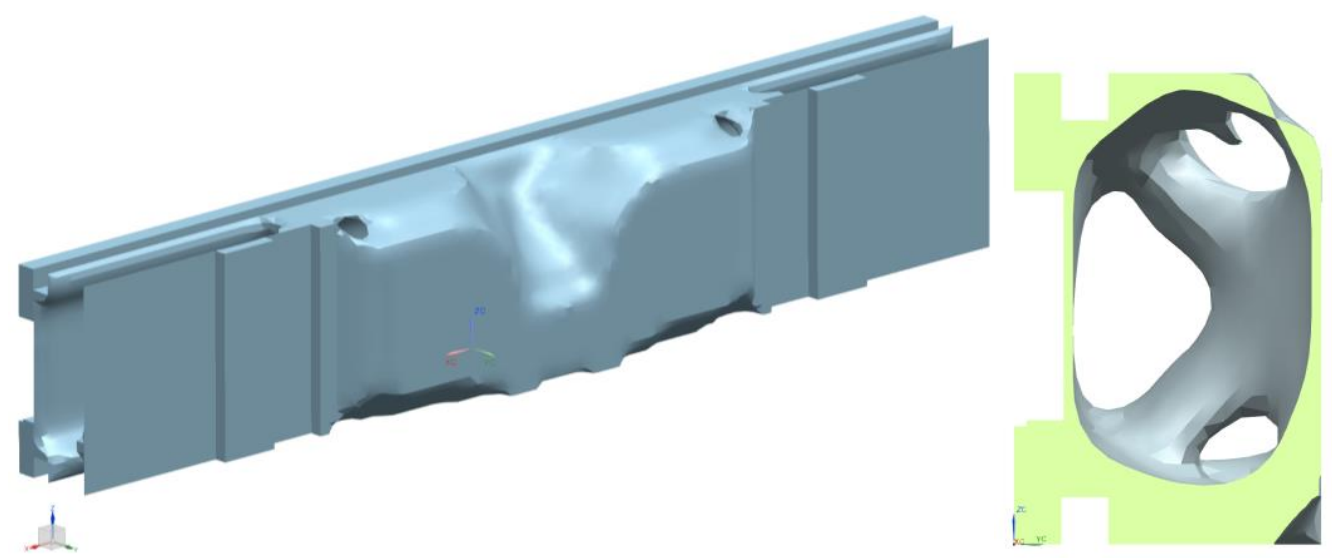

Fig. 1. Results of optimization with limited compliance in the guidance position 


\subsection{Consequences of guidance definition}

Definition of FEM model, definition of constrain and real load cases influence the topology-shape evolution. Stiffness of guidance influent material growth in the guidance area. Fig. 2 shows four states.

Guidance defined by rigid point-to-face connection by RBE2 elements reinforced virtual hydrostatic connection and influenced the solution. On one hand it causes distribution of stress and conservation of material in the guidance position. On the other hand it causes decrease of material mass in the center of the crossbeam, one can see in the Fig. 1. Partial compliance in the guidance definition with RBE3 elements of CBUSH elements with defined stiffness causes the decrease of virtual density in the guidance position as can be seen in the Fig. 2.

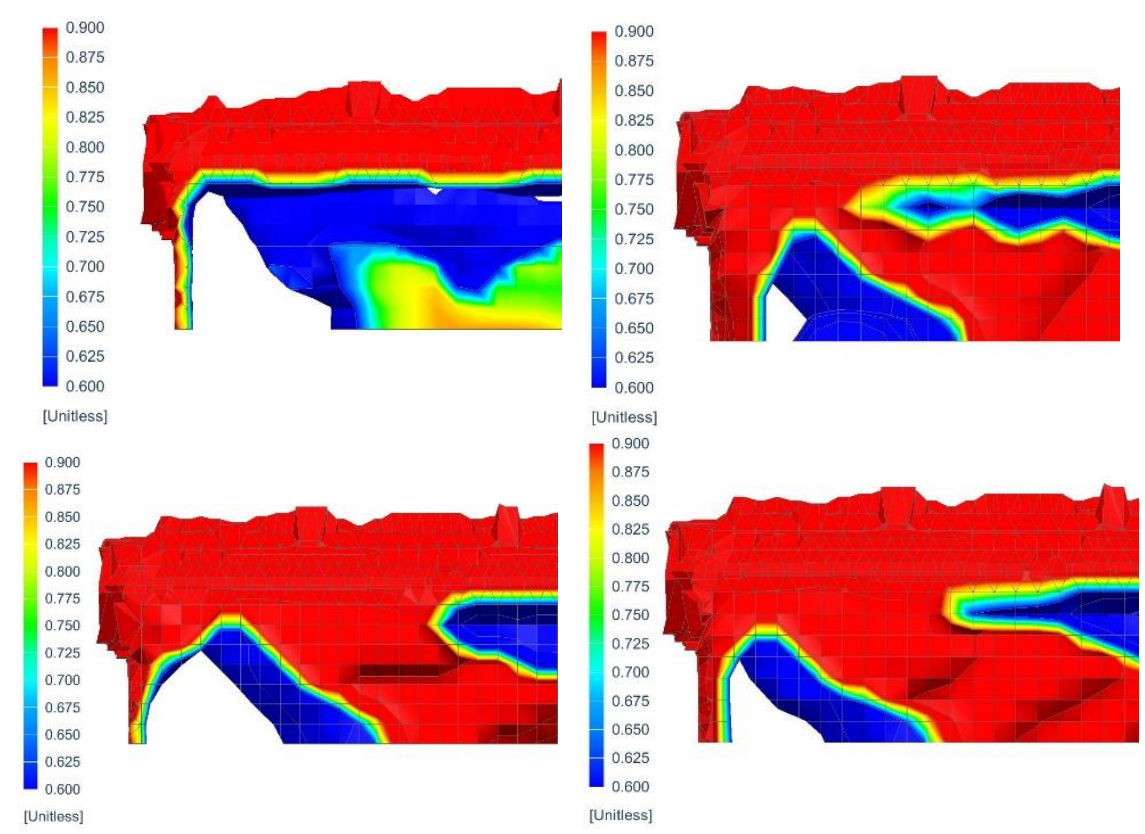

Fig. 2. Reinforcement based on constrain definition

\subsection{Manufacturing constrains}

The mirror manufacturing constrains is set into the simulation. It causes symmetrical growth of structure. Symmetrical requirement influences processing after the sensitivity study. Results of the calculation without symmetry requirement can be seen in the Fig. 3.

Minimal-element-size function restricts the minimal size of the generated material growth. This restriction requires specific quality of mesh. Mesh size has to be smaller than required minimal element size. Fine mesh means higher computation requirements. This manufacturing constrain may lead to results which are possible to be directly produced by additive methods. Conventional methods of casting require post-processing and design adjustment of topologyproduced results.

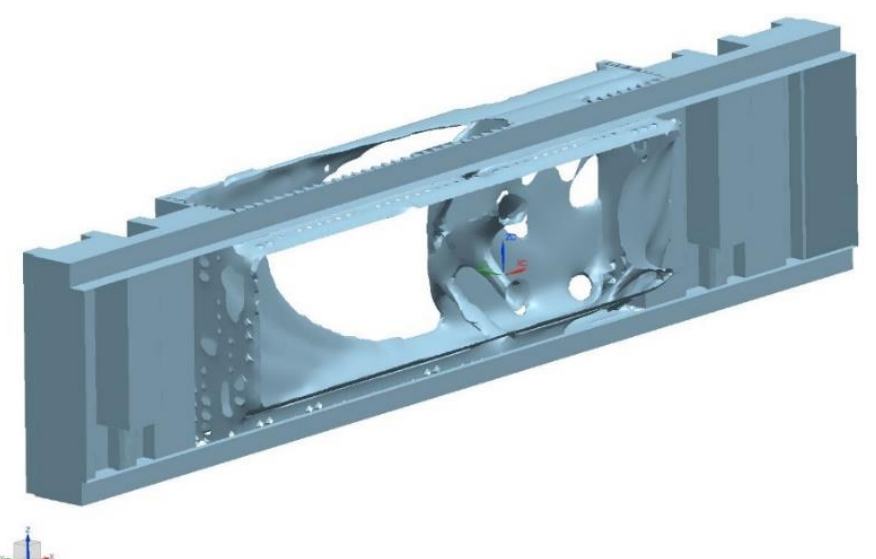

t.

Fig. 3. Section view into the crossbeam, loaded by asymmetric load force, no symmetric manufacturing constrain 
Reinforcement in the centre area is caused by the definition of constrain. Stiff structure which can be seen in the Fig. 4 on the right side is caused by compliant fixation in the vertical guidance. Structure represented on the left side is defined by stiff connection in the vertical guidance.

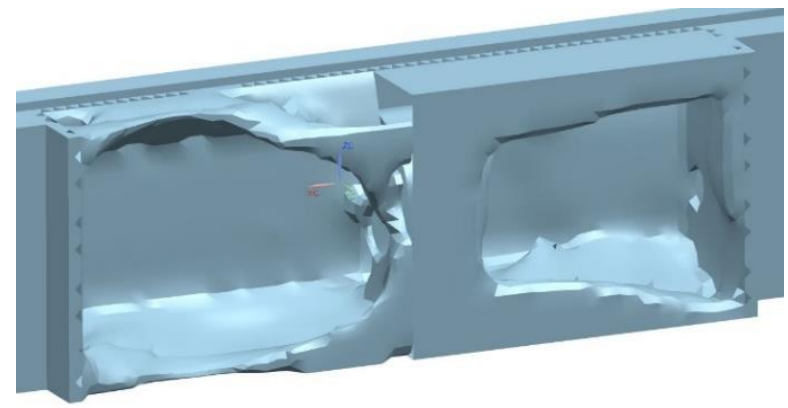

Fig. 4. Center area of crossbeam, reinforcement with respect to overall weigh and the X-fixation

\section{Evolution of welded structure}

Results of the topology optimization have to be adjusted for the design of welded structure. Main character is retained. Topology-shape needs an interpretation by the conventional ribs which is done by skilled engineer. Interpretation of the topology optimization with ribs can be seen in the Fig. 5. Equivalent problem is solved by Rinku [17]. Structure made of sheet ribs cannot reproduce topology structure, but main character can be followed. Thickness of designed sheets also varies due to topology. Presented weld study consist of 30/40/50mm thick sheets.

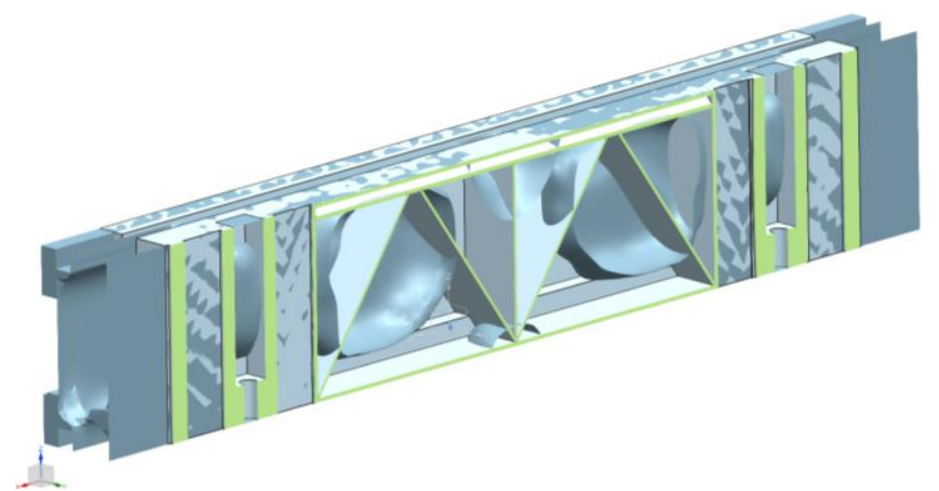

Fig. 5. Design with ribs - section view

\section{Preload}

Preloaded construction is often used for heavy loaded parts where unnecessary weight causes additive loads. Topology optimization identifies suitable positions of preload, which can reduce specific deformation. Material flow is replaced by pre-loaded $1 \mathrm{D}$ feature. Preload is defined to be $100 \mathrm{kN}$ in lower position and $150 \mathrm{kN}$ in upper position. Preload decreases vertical deformation of crossbeam $<0.04 \mathrm{~mm}$ total deformation in the tool position is approx. $0.1 \mathrm{~mm}$. Simulation of static load is defined by the boundary condition of cutting force and position. Observed deformation of preloaded part is decreased almost to $50 \%$.

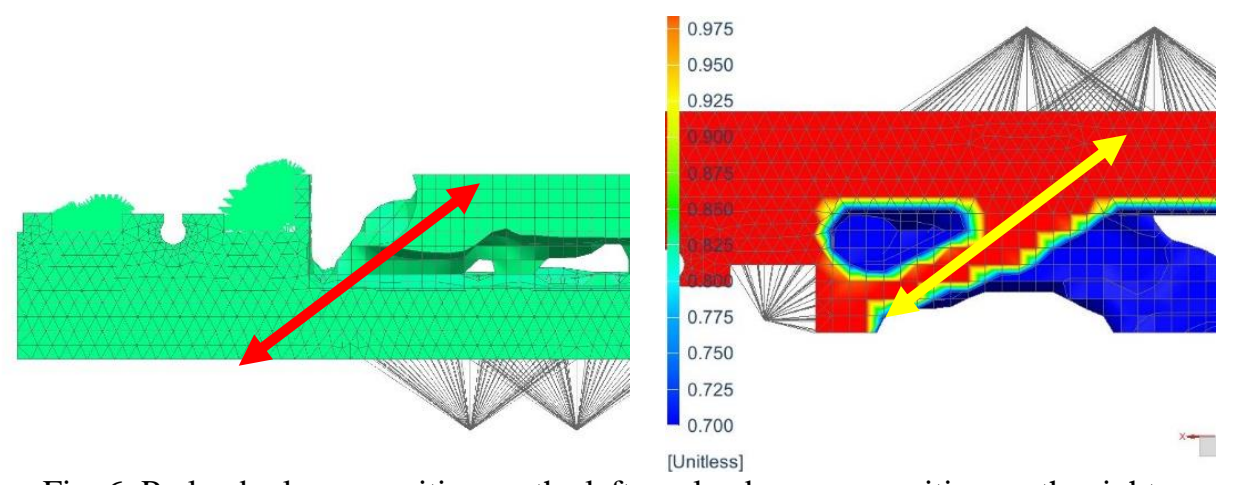

Fig. 6. Preload - lower position on the left, preload - upper position on the right 


\section{Results}

Final stiffness of structure is verified by Nastran linear solver (SOL101). Structure made of sheets based on the topology results was designed and calculated. Results show a good distribution of stress and suitable deformation. Comparison in the figure bellowFig. 7 shows option. Advanced reinforcement of non-preloaded structure leads to heavier structure which is more loaded by its own mass. Results of preloaded structure indicate the advantages of solution supported by topology optimization. One of the disadvantages is extra deformation in preloaded areas. Magnitude of deformation of top guidance edge can be seen in the graphs below the graphics. Areas of preload effect must be well designed for good stress distribution. It influents critical preload conditions, value of preload force for example. It should be emphasized that angular deformations of preloaded parts are dramatically lower than deformation of part without preload.

Deformation of top edge of preloaded crossbeam is less than $0.05 \mathrm{~mm}$. It is suitable for tool positioning and also for hydrostatic guidance. Tool position signs magnitude deformation $0.15 \mathrm{~mm}$ which is suitable for this critical condition with full cutting load. Observed stress is lower than 5Mpa, due to deformation-focused design. Area of preload effect is loaded by significantly higher stress. Final design of this area have to by optimized with respect to preload principle and option possibilities (preload generation, assembly adjustment etc.)

$$
\begin{aligned}
& 0.1000 \\
& 0.0917 \\
& 0.0833 \\
& 0.0750 \\
& 0.0667 \\
& 0.0583 \\
& 0.0500 \\
& 0.0417 \\
& 0.0333 \\
& 0.0250 \\
& 0.0167 \\
& 0.0083 \\
& 0.0000 \\
& {[\mathrm{~mm}]}
\end{aligned}
$$

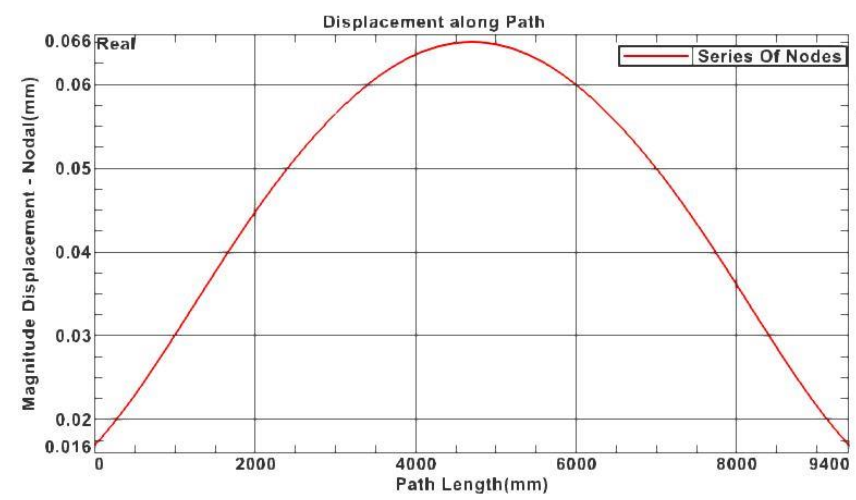

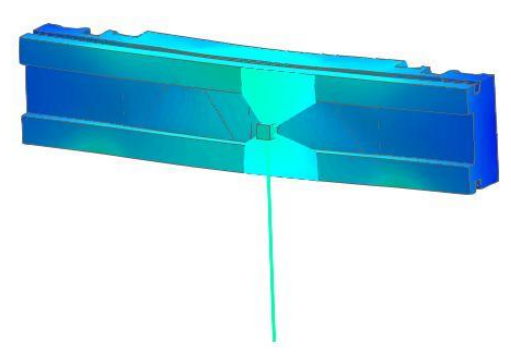

$[\mathrm{mm}]$

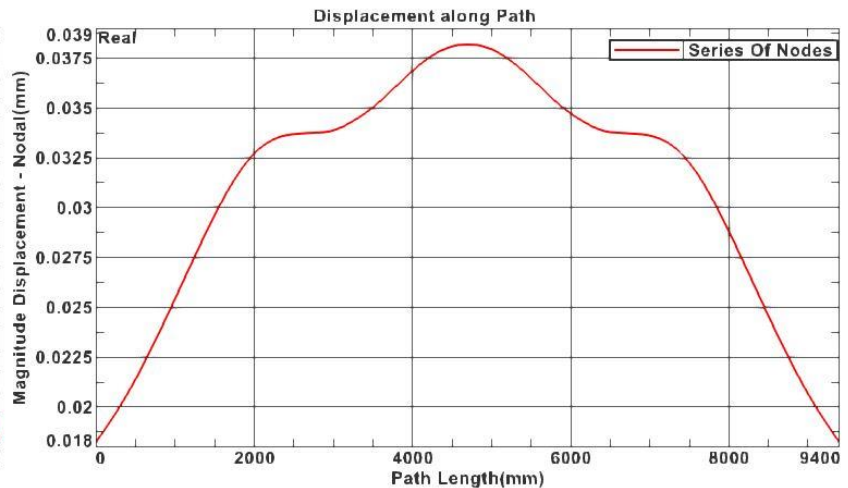

Fig. 7. Comparison of non-preloaded part (left) and preloaded (right) with deformation of top edge in the graph

\section{Conclusion}

The simulation and the prediction of deformation in the machine tools are a high value source of information. Methodology of optimization of developed portal structure was stated. This consists of definition of boundary conditions, topology setup and results interpretation. Topology optimization leads to good utilization of material with respect to boundary condition and loading cases. Final weight requirement was fulfilled. Described solution is strongly requested by machine tool producers. Approach in this area increases optimization of the thermal behaviour and structural properties of the machine tools. Developed loaded structure sign suitable levels of deformation and allow preload option which substantially decrease deformation. This allows an increase of the overall machining performance. Described progress also induces request of development in affiliated parts of the system. Development of all new machines requires complex approach or precise specification of connection between subsystems. Well described boundary conditions and loading cases were emphasised due to their vital influences on the results. 


\section{Acknowledgement}

The project LO1502 'Development of the Regional Technological Institute is carried out under the auspices of the National Sustainability Program I of the Ministry of Education of the Czech Republic

\section{References}

[1] Olason, Anton a Daniel Tidman. Methodology for Topology and Shape Optimization in the Design Process. Göteborg: Chalmers univerzity of technology, 2010.

[2] Johan Henrik, Martens, Zwick Daniel a Muskulus Michael. Topology Optimization of a Jacket Structure for an Offshore Wind Turbine with a Genetic Algorithm. In: 11th World Congress on Structural and Multidisciplinary Optimization. Sydney, 2015, s. 780-785.

[3] Wook-Han, Choi, Huang Cheng-Guo, Kim Jong-Moon a Park Gyung-Jin. Comparison of some commercial software systems for structural optimization. In: 11th World Congress on Structural and Multidisciplinary Optimisation. Sydney, 2015, s. 71-76.

[4] Ling, Zhao, Ma Jianfeng a Hongliang, Wuyi Chen. Lightweight Design and Verification of Gantry Machining Center Crossbeam Based on Structural Bionics. Jilin: Jilin University, 2011. DOI: 10.1016/S1672-6529(11)600218 .

[5] Wang-Chih, Chena A Chen Jahau Lewis. Eco-innovation by Integrating Biomimetic Design and ARIZ. 21st CIRP Conference on Life Cycle Engineering. Tainan: National Cheng Kung Unibversity, TAIWAN, 2014. DOI: 10.1016/j.procir.2014.06.028.

[6] Ling, Zhao, Ma Jianfeng, Wang Ting a Xing Denghai. Lightweight Design of Mechanical Structures based on Structural Bionic Methodology. Journal of Bionic Engineering. Elsevier Limited and Science Press, 2010. DOI: 10.1016/S1672-6529(09)60239-0.

[7] Danny, Lohan, Dede Ercan a Allison James. Topology Optimization for Heat Conduction Using Generative Design Algorithms. In: 11th World Congress on Structural and Multidisciplinary Optimization11th World Congress on Structural and Multidisciplinary Optimization. Sydney, 2015, s. 304-309.

[8] Hans-Christian, Mohring, Brecher Christian, Abele Eberhard, Fleischer Jurgen a Bleicher Friedrich. Materials in machine tool structures. Manufacturing Technology. CIRP Annals, 2015, s. 725-748.

[9] Marek, Vaclav. Sensitivity analysis and validation of a thermo-mechanical model of a spindle. In: Annals of DAAAM and Proceedings of the International DAAAM Symposium. Zadar, 2018, s. 703-708.

[10] Janda, Petr a Roman Polák. Virtual Prototyping and Optimization Of Heavy Machine Tools. In: Proceedings of the 26th DAAAM International Symposium. Vienna: DAAAM International, 2016, s. 967-973.

[11] Ráž, Karel, M Cechura a Zdeněk Chval. Upper Crossbeam of Free Forging Press Optimization in Order to Improve Mechanical Properties and Durability. In: Annals of DAAAM for 2015. Vienna: Danube Adria Association for Automation and Manufacturing, 2015, s. 524-529.

[12] M.P., Bendsoe a Sigmund O. Topology optimization : theory and applications. Berlin: Springer, 2013.

[13] Camelia Elena, Munteanu a Cismilianu Alexandru-Mihai. Structural Optimization of Space Components Adapted For 3D Printing. In: Annals of DAAAM and Proceedings of the International DAAAM Symposium. Zadar: DAAAM International, 2017, s. 0821-0825.

[14] Park, Hong a Prakash Dahal. Development of Topology Optimized Cable Joint System for Oil Storage Tanks. In: Annals of DAAAM for 2012 \& Proceedings of the 23rd International DAAAM Symposium. Vienna, 2012, 23, s. 409-412.

[15] FE-Design. Simulia Tosca Structure Documentation 8.1. Karlsruhe: FE-DESIGN GmbH, 2014.

[16] Carl-Johan, Thore, Holmberg Erik a Klarbring Anders. Large-scale robust topology optimization under loaduncertainty. In: 11th World Congress on Structural and Multidisciplinary Optimization. Sydney, 2015, s. 255-260.

[17] A, Rinku a Ananthasuresh G.K. Topology and Size Optimization of Modular Ribs in Aircraft Wings. In: 11th World Congress on Structural and Multidisciplinary Optimisation. Sydney, 2015. 